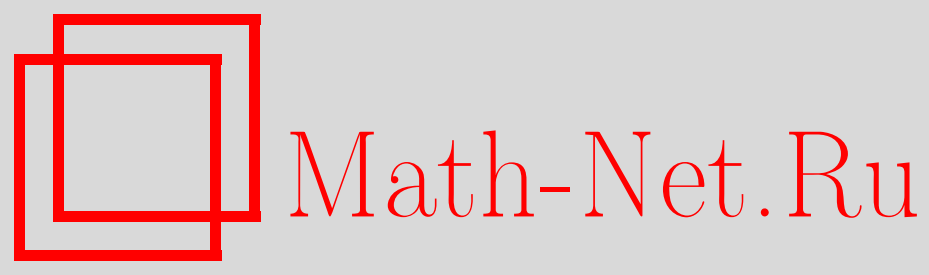

И. В. Шрагин, О некоторых $\sigma$-алгебрах, связанных с измеримостью суперпозиций, Матем. заметки, 2006, том 80, выпуск 6, 926-933

DOI: https://doi.org/10.4213/mzm3368

Использование Общероссийского математического портала Math-Net.Ru подразумевает, что вы прочитали и согласны с пользовательским соглашением http://www . mathnet.ru/rus/agreement

Параметры загрузки:

IP : 18.209 .158 .208

26 апреля 2023 г., 08:32:12

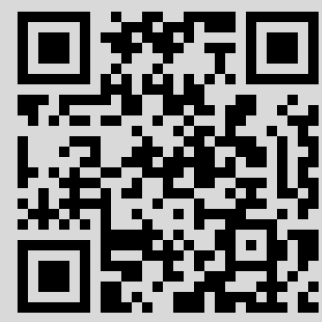




\section{О НЕКОТОРЫХ $\sigma$-АЛГЕБРАХ, СВЯЗАННЫХ С ИЗМЕРИМОСТЬЮ СУПЕРПОЗИЦИЙ}

\section{И. В. Шрагин}

Даны измеримое пространство $(T, \mathscr{T})$, множество $X$ и функция $\varphi: T \rightarrow X$. Рассматриваются $\sigma$-алгебры

$$
\mathfrak{N}_{\varphi}=\left\{B \subset X: \varphi^{-1}(B) \in \mathscr{T}\right\} \quad \text { и } \quad \mathfrak{M}_{\varphi}=\left\{D \subset T \times X: G_{\varphi}^{-1}(D) \in \mathscr{T}\right\},
$$

где $G_{\varphi}(t)=(t, \varphi(t))$, а также $\mathfrak{N}_{\Phi}=\bigcap_{\varphi \in \Phi} \mathfrak{N}_{\varphi}$ и $\mathfrak{M}_{\Phi}=\bigcap_{\varphi \in \Phi} \mathfrak{M}_{\varphi}$, где $\Phi \subset X^{T}$. Эти $\sigma$-алгебры фигурируют в критериях $(\mathscr{T}, \mathscr{B})$-измеримости суперпозиций $g \circ \varphi$ и $f \circ G_{\varphi}$ с функциями $g: X \rightarrow Y$ и $f: T \times X \rightarrow Y$, где $(Y, \mathscr{B})$ - измеримое пространство. В работе дано описание элементов указанных $\sigma$-алгебр, не использующее операций $\varphi^{-1}$ и $G_{\varphi}^{-1}$.

Библиография: 4 названия.

Введение. Пусть $(T, \mathscr{T})$ - измеримое пространство, а $X$ - непустое множество. Для данной функции $\varphi: T \rightarrow X$ определим ее "график-функцию" $G_{\varphi}: T \rightarrow T \times X$, где $G_{\varphi}(t)=(t, \varphi(t)), t \in T$ (так что $G_{\varphi}(T)=\operatorname{gr} \varphi-$ график функции $\left.\varphi\right)$. С помощью функции $G_{\varphi}$ и $\sigma$-алгебры $\mathscr{T}$ определим $\sigma$-алгебру $\mathfrak{M}_{\varphi}$ на декартовом произведении $T \times X:$

$$
\mathfrak{M}_{\varphi}:=\left\{D \subset T \times X: G_{\varphi}^{-1}(D) \in \mathscr{T}\right\}
$$

(символом ":=" обозначается определяющее равенство). $\sigma$-алгебра $\mathfrak{M}_{\varphi}$ фигурирует в следующем критерии измеримости суперпозиции $f \circ G_{\varphi}: T \rightarrow Y$, где $(Y, \mathscr{B})-$ измеримое пространство, а $f: T \times X \rightarrow Y$ - некоторая функция.

КРИТЕРИЙ ИЗмЕРИмОСТИ СУПЕРПОЗИЦИИ. Суперпозииия $f \circ G_{\varphi}$ является $(\mathscr{T}$, $\mathscr{B})$-измеримой, m.e.

$$
(\forall C \in \mathscr{B}) \quad\left(f \circ G_{\varphi}\right)^{-1}(C) \in \mathscr{T},
$$

тогда и только тогда, когда функиия $f$ является $\left(\mathfrak{M}_{\varphi}, \mathscr{B}\right)$-измеримой.

Это утверждение непосредственно следует из равенства

$$
\left(f \circ G_{\varphi}\right)^{-1}(C)=G_{\varphi}^{-1}\left[f^{-1}(C)\right], \quad C \in \mathscr{B},
$$

и определения $\sigma$-алгебры $\mathfrak{M}_{\varphi}$.

Далее, пусть $\Phi-$ некоторое множество функций $\varphi: T \rightarrow X$. Тогда, как очевидно, суперпозиция $f \circ G_{\varphi}(\mathscr{T}, \mathscr{B})$-измерима для любой функции $\varphi \in \Phi$ в том и только том случае, когда функция $f\left(\mathfrak{M}_{\Phi}, \mathscr{B}\right)$-измерима, где

$$
\mathfrak{M}_{\Phi}:=\left\{D \subset T \times X:(\forall \varphi \in \Phi), \quad G_{\varphi}^{-1}(D) \in \mathscr{T}\right\}
$$

(С) И.В. ШРАГИН, 2006 
т.е. $\mathfrak{M}_{\Phi}=\bigcap_{\varphi \in \Phi} \mathfrak{M}_{\Phi}$.

Изложенные положения по существу содержатся в [1] и [2] (см. также [3; с. 1819]). Однако до сих пор оставалось неизвестным явное описание элементов $\sigma$-алгебр $\mathfrak{M}_{\varphi}$ и $\mathfrak{M}_{\Phi}$, не использующее операцию $G_{\Phi}^{-1}$. Эта проблема решается в настоящей работе путем разбиения $\sigma$-алгебры $\mathfrak{M}_{\varphi}$ на дизъюнктные классы $K(\varphi, A), A \in \mathscr{T}$, допускающие простое описание их элементов.

Дано также описание элементов $\sigma$-алгебр

$$
\mathfrak{N}_{\varphi}:=\left\{B \subset X: \varphi^{-1}(B) \in \mathscr{T}\right\}
$$

и $\mathfrak{N}_{\Phi}:=\bigcap_{\varphi \in \Phi} \mathfrak{N}_{\varphi}$, которые связаны с $(\mathscr{T}, \mathscr{B})$-измеримостью суперпозиций вида $g \circ \varphi: T \rightarrow Y$, образованных с помощью функции $g: X \rightarrow Y$.

Отметим, что мы употребляем символ вложения множеств "С" и в случае их равенства.

1. Описание элементов $\sigma$-алгебр $\mathfrak{M}_{\varphi}$ и $\mathfrak{M}_{\Phi}$. Итак, нам даны измеримое пространство $(T, \mathscr{T})$, непустое множество $X$ и функция $\varphi: T \rightarrow X$. Положим при $A \in \mathscr{T}$

$$
\mathscr{K}(\varphi, A):=\left\{D \subset T \times X: G_{\varphi}^{-1}(D)=A\right\} .
$$

Тогда, как очевидно,

$$
\mathfrak{M}_{\varphi}=\bigcup_{A \in \mathscr{T}} \mathscr{K}(\varphi, A) .
$$

Ясно, что если $A_{1} \neq A_{2}$, то $\mathscr{K}\left(\varphi, A_{1}\right) \cap \mathscr{K}\left(\varphi, A_{2}\right)=\varnothing$. Таким образом, $\sigma$-алгебра $\mathfrak{M}_{\varphi}$ разбита на дизъюнктные классы $\mathscr{K}(\varphi, A)$, соответствующие всевозможным множествам $A \in \mathscr{T}$.

Очевидно, при данном $A$ семейство $\mathscr{K}(\varphi, A)$ содержит любые объединения и пересечения входящих в него множеств. Кроме того, оно "выпукло" в том смысле, что если $D_{1} \subset D_{2}$, где $D_{i} \in \mathscr{K}(\varphi, A), i=1,2$, и $D_{1} \subset D \subset D_{2}$, то $D \in \mathscr{K}(\varphi, A)$.

Следующая теорема дает явное описание (т.е. без помощи операции $G_{\varphi}^{-1}$ ) элементов класса $\mathscr{K}(\varphi, A)$.

Tеорема 1. При любом $A \in \mathscr{T}$

$$
\mathscr{K}(\varphi, A)=\left\{D \subset T \times X: \operatorname{gr} \varphi \cap(A \times X) \subset D \subset D_{A}\right\},
$$

где

$$
D_{A}=(A \times X) \cup\{[(T \backslash A) \times X] \backslash \operatorname{gr} \varphi\} .
$$

ДокАЗАТЕЛЬСтво. Так как

$$
G_{\varphi}^{-1}(\operatorname{gr} \varphi \cap(A \times X))=T \cap A=A,
$$

то $\operatorname{gr} \varphi \cap(A \times X) \in \mathscr{K}(\varphi, A)$. Далее, пусть $D \in \mathscr{K}(\varphi, A)$, т.е. $G_{\varphi}^{-1}(D)=A$. Тогда если $(t, x) \in \operatorname{gr} \varphi \cap(A \times X)$, то $x=\varphi(t)$, причем $t \in A$. Значит, $(t, \varphi(t)) \in D$, т.е. $(t, x) \in D$. Таким образом, gr $\varphi \cap(A \times X) \subset D$, т.е. множество $\operatorname{gr} \varphi \cap(A \times X)$ является минимальным (в смысле включения) в классе $\mathscr{K}(\varphi, A)$.

Покажем, что множество $D_{A}$ максимально в $\mathscr{K}(\varphi, A)$. В самом деле, так как

$$
G_{\varphi}^{-1}\{[(T \backslash A) \times X] \backslash \operatorname{gr} \varphi\}=\varnothing,
$$


то $G_{\varphi}^{-1}\left(D_{A}\right)=G_{\varphi}^{-1}(A \times X)=A$, т.е. $D_{A} \in \mathscr{K}(\varphi, A)$. Возьмем теперь произвольное $D \in \mathscr{K}(\varphi, A)$ и покажем, что $D \subset D_{A}$. Для этого достаточно показать, что

$$
D \backslash(A \times X) \subset[(T \backslash A) \times X] \backslash \operatorname{gr} \varphi .
$$

Итак, пусть $(t, x) \in D \backslash(A \times X)$. Тогда $(t, x) \notin \operatorname{gr} \varphi$, так как иначе $x=\varphi(t)$, откуда $G_{\varphi}(t)=(t, x) \in D$, т.е. $t \in G_{\varphi}^{-1}(D)=A$ - противоречие. Тем самым доказано включение (3), так что $D \subset D_{A}$.

Остается применить отмеченную выше "выпуклость" класса $\mathscr{K}(\varphi, A)$.

Перейдем к $\sigma$-алгебре $\mathfrak{M}_{\Phi}$ при некотором $\Phi \subset X^{T}$. Так как $\mathfrak{M}_{\Phi}=\bigcap_{\varphi \in \Phi} \mathfrak{M}_{\varphi}$, то в виду (1)

$$
\mathfrak{M}_{\Phi}=\bigcap_{\varphi \in \Phi} \bigcup_{A \in \mathscr{T}} \mathscr{K}(\varphi, A) .
$$

Однако для $\mathfrak{M}_{\Phi}$ существует более удобное представление, а именно

$$
\mathfrak{M}_{\Phi}=\bigcap_{F \in \mathscr{T}^{\Phi}} \bigcap_{\varphi \in \Phi} \mathscr{K}(\varphi, F(\varphi)) .
$$

Действительно, пусть $D \in \mathfrak{M}_{\Phi}$, т.е.

$$
(\forall \varphi \in \Phi) \quad G_{\varphi}^{-1}(D) \in \mathscr{T} .
$$

Определим отображение $F_{D}: \Phi \rightarrow \mathscr{T}$, положив $F_{D}(\varphi)=G_{\varphi}^{-1}(D), \varphi \in \Phi$. Значит,

$$
(\forall \varphi \in \Phi) \quad D \in \mathscr{K}\left(\varphi, F_{D}(\varphi)\right)
$$

так что $D$ принадлежит правой части равенства (4).

Обратно, если

$$
\left(\exists F \in \mathscr{T}^{\Phi} \quad(\forall \varphi \in \Phi) \quad D \in \mathscr{K}(\varphi, F(\varphi)),\right.
$$

то

$$
(\forall \varphi \in \Phi) \quad G_{\varphi}^{-1}(D)=F(\varphi) \in \mathscr{T}
$$

т.е. $D \in \mathfrak{M}_{\Phi}$.

Тем самым, равенство (4) доказано. Заметим, что из дизъюнктности классов $\mathscr{K}(\varphi, A)$ при различных $A \in \mathscr{T}$ следует дизъюнктность семейств $\bigcap_{\varphi \in \Phi} \mathscr{K}(\varphi, F(\varphi))$ при различных $F \in \mathscr{T}^{\Phi}$.

Таким образом, равенство (4) представляет собой разложение $\sigma$-алгебры $\mathfrak{M}_{\Phi}$ на дизъюнктные семейства подмножеств множества $T \times X$.

Представим теперь $\bigcap_{\varphi \in \Phi} \mathscr{K}(\varphi, F(\varphi))$ при данном отображении $F: \Phi \rightarrow \mathscr{T}$ в более обозримом виде, что позволит, кстати, получить условие непустоты этого пересечения. А именно, в силу (2)

$$
\begin{aligned}
\bigcap_{\varphi \in \Phi} \mathscr{K}(\varphi, F(\varphi)) & =\bigcap_{\varphi \in \Phi}\left\{D: \operatorname{gr} \varphi \cap(F(\varphi) \times X) \subset D \subset D_{F(\varphi)}\right\} \\
& =\left\{D: \bigcup_{\varphi \in \Phi}[\operatorname{gr} \varphi \cap(F(\varphi) \times X)] \subset D \subset \bigcap_{\psi \in \Phi} D_{F(\psi)}\right\},
\end{aligned}
$$

где

$$
D_{F(\psi)}=(F(\psi) \times X) \cup\{[(T \backslash F(\psi)) \times X] \backslash \operatorname{gr} \psi\} .
$$


Отсюда следует, что $\bigcap_{\varphi \in \Phi} \mathscr{K}(\varphi, F(\varphi)) \neq \varnothing$ тогда и только тогда, когда при любых $\varphi, \psi \in \Phi$

$$
\operatorname{gr} \varphi \cap(F(\varphi) \times X) \subset D_{F(\psi)} .
$$

В частности, если отображение $F_{0}$ - "константа", т.е.

$$
(\forall \varphi \in \Phi) \quad F_{0}(\varphi)=A_{0} \in \mathscr{T},
$$

то $\bigcap_{\varphi \in \Phi} \mathscr{K}\left(\varphi, F_{0}(\varphi)\right) \neq \varnothing$, так как

$$
\forall(\varphi, \psi \in \Phi) \quad \operatorname{gr} \varphi \cap\left(F_{0}(\varphi) \times X\right) \subset A_{0} \times X \subset D_{F_{0}(\psi)} .
$$

Рассмотрим в качестве примера случай тривиальной $\sigma$-алгебры $\mathscr{T}=\{\varnothing, T\}$. Тогда так как $\mathfrak{M}_{\varphi}=\mathscr{K}(\varphi, \varnothing) \cup \mathscr{K}(\varphi, T)$, то

$$
\mathfrak{M}_{\varphi}=\{D: D \cap \operatorname{gr} \varphi=\varnothing\} \cup\{D: \operatorname{gr} \varphi \subset D\} .
$$

Далее, пусть $F \in \mathscr{T}^{\Phi}$. Положим

$$
\Psi=\{\varphi \in \Phi: F(\varphi)=T\},
$$

так что $\Phi \backslash \Psi=\{\varphi \in \Phi: F(\varphi)=\varnothing\}$. Тогда

$$
\bigcap_{\varphi \in \Phi} \mathscr{K}(\varphi, F(\varphi))=\bigcap_{\varphi \in \Psi}\{D: \operatorname{gr} \varphi \subset D\} \cap \bigcap_{\varphi \in \Phi \backslash \Psi}\{D: D \cap \operatorname{gr} \varphi=\varnothing\},
$$

откуда

$$
\bigcap_{\varphi \in \Phi} \mathscr{K}(\varphi, F(\varphi))=\left\{D: \bigcap_{\varphi \in \Psi} \operatorname{gr} \varphi \subset D \subset(T \times X) \backslash \bigcup_{\varphi \in \Phi \backslash \Psi} \operatorname{gr} \varphi\right\} .
$$

Отсюда следует, что $\bigcap_{\varphi \in \Phi} \mathscr{K}(\varphi, F(\varphi)) \neq \varnothing$ тогда и только тогда, когда

$$
\forall(\psi \in \Psi, \varphi \in \Phi \backslash \Psi) \quad \operatorname{gr} \psi \cap \operatorname{gr} \varphi=\varnothing .
$$

Очевидно, равенство (6) определяет (в ситуации, когда $\mathscr{T}=\{\varnothing, T\}$ ) биекцию между отображениями $F \in \mathscr{T}^{\Phi}$ и подмножествами $\Psi \subset \Phi$. Поэтому в силу (4) и (6)

$$
\mathfrak{M}_{\Phi}=\bigcup_{\Psi \subset \Phi}\left\{D: \bigcup_{\varphi \in \Psi} \operatorname{gr} \varphi \subset D \subset(T \times X) \backslash \bigcup_{\varphi \in \Phi \backslash \Psi} \operatorname{gr} \varphi\right\} .
$$

Ясно, что в этом представлении $\sigma$-алгебры $\mathfrak{M}_{\Phi}$ фактически присутствуют только те подмножества $\Psi$, которые удовлетворяют условию (7).

2. Описание элементов $\sigma$-алгебр $\mathfrak{N}_{\varphi}$ и $\mathfrak{N}_{\Phi}$. Здесь, как и в п. 1 , даны измеримое пространство $(T, \mathscr{T})$ и непустое множество $X$. Для функции $\varphi: T \times X$ определим $\sigma$-алгебру

$$
\mathfrak{N}_{\varphi}:=\left\{B \subset X: \varphi^{-1}(B) \in \mathscr{T}\right\} .
$$

Если дана функция $g: X \rightarrow Y$, где $(Y, \mathscr{B})$ - измеримое пространство, то суперпозиция $g \circ \varphi: T \rightarrow Y$ является $(\mathscr{T}, \mathscr{B})$-измеримой тогда и только тогда, когда функция $g$ $\left(\mathfrak{N}_{\varphi}, \mathscr{B}\right)$-измерима. Это утверждение вытекает из равенства

$$
(g \circ \varphi)^{-1}(C)=\varphi^{-1}\left(g^{-1}(C)\right), \quad C \in \mathscr{B},
$$


и определения $\sigma$-алгебры $\mathfrak{N}_{\varphi}$.

Положим при $A \subset T$

$$
\mathscr{L}(\varphi, A)=\left\{B \subset X: \varphi^{-1}(B)=A\right\} .
$$

Очевидно,

$$
\mathfrak{N}_{\varphi}=\bigcup_{A \in \mathscr{T}} \mathscr{L}(\varphi, A)
$$

причем при различных $A$ классы $\mathscr{L}(\varphi, A)$ попарно не пересекаются. Однако в отличие от $\mathscr{K}(\varphi, A)$ класс $\mathscr{L}(\varphi, A)$ может быть пустым.

Лемма 1. При заданных функиии $\varphi: T \rightarrow X$ и множестве $A \subset T$ следующие условия попарно эквивалентны:

1) $\mathscr{L}(\varphi, A) \neq \varnothing$

2) $\varphi(A) \cap \varphi(T \backslash A)=\varnothing$

3) $\varphi^{-1}(\varphi(A))=A$.

Доказательство. Пусть $\mathscr{L}(\varphi, A) \neq \varnothing$, т.е. $\varphi^{-1}(B)=A$ при некотором $B \subset X$. Предположим, что условие 2) не выполняется. Тогда $\varphi(s)=\varphi(t)$ при некоторых $s \in$ $A$ и $t \in T \backslash A$. Так как $\varphi^{-1}(B)=A$, то $\varphi(s) \in B$, откуда и $\varphi(t) \in B$. Следовательно, $t \in \varphi^{-1}(B)$, т.е. $t \in A$. Полученное противоречие показывает, что из 1$)$ следует 2$)$.

Пусть выполняется условие 2). Возьмем $t \in \varphi^{-1}(\varphi(A))$, т.е. $\varphi(t) \in \varphi(A)$. Отсюда следует, что $t \in A$, так как в противном случае $\varphi(t) \in \varphi(T \backslash A)$, что противоречит условию 2). Таким образом, $\varphi^{-1}(\varphi(A)) \subset A$. Так как и обратное вложение имеет место, то условие 3) доказано.

Наконец, из 3) следует, что $\varphi(A) \in \mathscr{L}(\varphi, A)$, т.е. $\mathscr{L}(\varphi, A) \neq \varnothing$.

Отметим, что к непустым классам относятся $\mathscr{L}(\varphi, \varnothing)$ и $\mathscr{L}(\varphi, T)$.

Положим при заданной функции $\varphi: T \rightarrow X$

$$
\mathscr{T}_{\varphi}=\{A \in \mathscr{T}: \varphi(A) \cap \varphi(T \backslash A)=\varnothing\} .
$$

Тогда

$$
\mathfrak{N}_{\varphi}=\bigcup_{A \in \mathscr{T}_{\varphi}} \mathscr{L}(\varphi, A),
$$

причем все классы $\mathscr{L}(\varphi, A)$ в этом равенстве непусты.

Нетрудно проверить, что $\mathscr{T}_{\varphi}$ является $\sigma$-алгеброй на $T$. В частности, если функция $\varphi$ тождественно постоянна, т.е. $(\forall t \in T) \quad \varphi(t)=x_{0}$, то $\mathscr{T}_{\varphi}=\{\varnothing, T\}$. При этом

$$
\mathscr{L}(\varphi, \varnothing)=\left\{B: x_{0} \notin B\right\}, \quad \mathscr{L}(\varphi, T)=\left\{B: x_{0} \in B\right\} .
$$

Если же $\varphi$ - инъекция, то, как очевидно, $\mathscr{T}_{\varphi}=\mathscr{T}$.

Следующая теорема описывает состав класса $\mathscr{L}(\varphi, A)$.

Теорема 2. При любом $A \in \mathscr{T}$

$$
\mathscr{L}(\varphi, A)=\{B \subset X: \varphi(A) \subset B \subset X \backslash \varphi(T \backslash A)\} .
$$

Доказательство. Если $A \in \mathscr{T} \backslash \mathscr{T}_{\varphi}$, то обе части равенства (8) пусты. Пусть $A \in \mathscr{T}_{\varphi}$. Тогда в силу леммы 1 (условие 3$\left.)\right) \varphi(A) \in \mathscr{L}(\varphi, A)$. Более того, если $B \in \mathscr{L}(\varphi, A)$, т.е. $\varphi^{-1}(B)=A$, то $\varphi(A) \subset B$. Следовательно, множество $\varphi(A)$ минимально в $\mathscr{L}(\varphi, A)$. 
Далее, так как $T \backslash A \in \mathscr{T}_{\varphi}$, то $\varphi^{-1}(X \backslash \varphi(T \backslash A))=T \backslash(T \backslash A)=A$, т.е. $X \backslash \varphi(T \backslash A) \in$ $\mathscr{L}(\varphi, A)$.

Покажем, что если $B \in \mathscr{L}(\varphi, A)$, то $B \subset X \backslash \varphi(T \backslash A)$. С этой целью возьмем $x \in B$ и предположим, что $x \in \varphi(T \backslash A)$, т.е. $x=\varphi(t)$ при некотором $t \in T \backslash A$. Но так как $\varphi(t)=x \in B$, то $t \in \varphi^{-1}(B)=A$. Полученное противоречие показывает, что $x \notin \varphi(T \backslash A)$, т.е. $B \subset X \backslash \varphi(T \backslash A)$. Следовательно, множество $X \backslash \varphi(T \backslash A)$ максимально в классе $\mathscr{L}(\varphi, A)$.

Остается заметить, что если $\varphi(A) \subset B \subset X \backslash \varphi(T \backslash A)$, то $B \in \mathscr{L}(\varphi, A)$ в силу свойства "выпуклости", которым класс $\mathscr{L}(\varphi, A)$ обладает аналогично классу $\mathscr{K}(\varphi, A)$.

Рассмотрим теперь $\sigma$-алгебру $\mathfrak{N}_{\Phi}$ и покажем, что

$$
\mathfrak{N}_{\Phi}=\bigcup_{F \in \mathscr{T}^{\Phi}} \bigcap_{\varphi \in \Phi} \mathscr{L}(\varphi, F(\varphi)) .
$$

Действительно, пусть $B \in \mathfrak{N}_{\Phi}$, т.е. $(\forall \varphi \in \Phi) \quad \varphi^{-1}(B) \in \mathscr{T}$. Положим $F_{B}(\varphi)=$ $\varphi^{-1}(B), \varphi \in \Phi$. Тогда $F_{B} \in \mathscr{T}^{\Phi}$ и $(\forall \varphi \in \Phi) \quad B \in \mathscr{L}\left(\varphi, F_{B}(\varphi)\right)$, т.е. $B$ принадлежит правой части (9). Обратно, если

$$
\left(\exists F \in \mathscr{T}^{\Phi}\right) \quad(\forall \varphi \in \Phi) \quad B \in \mathscr{L}(\varphi, F(\varphi)),
$$

TO

$$
(\forall \varphi \in \Phi) \quad \varphi^{-1}(B)=F(\varphi) \in \mathscr{T},
$$

т.е. $B \in \mathfrak{N}_{\Phi}$.

Очевидно, равенство (9) представляет собой разложение $\sigma$-алгебры $\mathfrak{N}_{\Phi}$ на дизъюнктные семейства подмножеств множества $X$. Выясним, при каких условиях эти семейства непусты.

Так как в силу (8) при фиксированном $F \in \mathscr{T}^{\Phi}$

$$
\mathscr{L}(\varphi, F(\varphi))=\{B \subset X: \varphi(F(\varphi)) \subset B \subset X \backslash \varphi(T \backslash F(\varphi))\},
$$

Tо

$$
\bigcap_{\varphi \in \Phi} \mathscr{L}(\varphi, F(\varphi))=\left\{B: \bigcup_{\varphi \in \Phi} \varphi(F(\varphi)) \subset B \subset X \backslash \bigcup_{\varphi \in \Phi} \varphi(T \backslash F(\varphi))\right\} .
$$

Отсюда следует, что $\bigcap_{\varphi \in \Phi} \mathscr{L}(\varphi, F(\varphi)) \neq \varnothing$ тогда и только тогда, когда

$$
\forall(\varphi, \psi \in \Phi) \quad \varphi(F(\varphi)) \cap \psi(T \backslash F(\psi))=\varnothing .
$$

Таким образом, в формуле (9) фактически присутствуют лишь те $F \in \mathscr{T}^{\Phi}$, которые удовлетворяют условию (10).

Как и в п. 1 , рассмотрим случай, когда $\mathscr{T}=\{\varnothing, T\}$. Так как

$$
\mathscr{L}(\varphi, \varnothing)=\{B: B \subset X \backslash \varphi(T)\}, \quad \mathscr{L}(\varphi, T)=\{B: \varphi(T) \subset B\},
$$

то

$$
\mathfrak{N}_{\varphi}=\{B: B \subset X \backslash \varphi(T)\} \cup\{B: \varphi(T) \subset B\} .
$$

Далее, пусть $\Phi \subset X^{T}$ и $F \in \mathscr{T}^{\Phi}$. Положим $\Psi=\{\varphi \in \Phi: F(\varphi)=T\}$. Тогда

$$
\begin{aligned}
\bigcap_{\varphi \in \Phi} \mathscr{L}(\varphi, F(\varphi)) & =\bigcap_{\varphi \in \Psi} \mathscr{L}(\varphi, T) \cap \bigcap_{\varphi \in \Phi \backslash \Psi} \mathscr{L}(\varphi, \varnothing) \\
& =\left\{B: \bigcap_{\varphi \in \Psi} \varphi(T) \subset B \subset X \backslash \bigcap_{\varphi \in \Phi \backslash \Psi} \varphi(T)\right\} .
\end{aligned}
$$


Отсюда следует, что $\bigcap_{\varphi \in \Phi} \mathscr{L}(\varphi, F(\varphi) \neq \varnothing$ тогда и только тогда, когда

$$
\forall(\psi \in \Psi, \varphi \in \Phi \backslash \Psi) \quad \varphi(T) \cap \psi(T)=\varnothing .
$$

Таким образом, если $\mathscr{T}=\{\varnothing, T\}$ и $\Phi \subset X^{T}$, то в силу (9)

$$
\mathfrak{N}_{\Phi}=\bigcup_{\psi \in \Phi}\left\{B: \bigcup_{\varphi \in \Psi} \varphi(T) \subset B \subset X \backslash \bigcup_{\varphi \in \Phi \backslash \Psi} \varphi(T)\right\} .
$$

При этом здесь можно ограничиться лишь теми $\Psi \subset \Phi$, для которых выполняется условие (11).

\section{3. Некоторые замечания.}

ЗАмечАниЕ 1 . Зафиксируем функцию $\varphi: T \rightarrow X$ и положим

$$
\mathscr{T} \times \mathfrak{N}_{\varphi}:=\left\{A \times B: A \in \mathscr{T}, B \in \mathfrak{N}_{\varphi}\right\} .
$$

Тогда $\mathscr{T} \times \mathfrak{N}_{\varphi} \subset \mathfrak{M}_{\varphi}$, так как если $A \in \mathscr{T}$, а $B \in \mathfrak{N}_{\varphi}$, то

$$
G_{\varphi}^{-1}(A \times B)=\{t:(t, \varphi(t)) \in A \times B\}=A \cap \varphi^{-1}(B) \in \mathscr{T} .
$$

Обозначим через $\mathscr{T} \otimes \mathfrak{N}_{\varphi} \sigma$-алгебру на $T \times X$, порожденную семейством $\mathscr{T} \times \mathfrak{N}_{\varphi}$. Так как $\mathfrak{M}_{\varphi}-\sigma$-алгебра, то

$$
\mathscr{T} \otimes \mathfrak{N}_{\varphi} \subset \mathfrak{M}_{\varphi}
$$

Покажем, что это вложение, вообще говоря, не является равенством. С этой целью рассмотрим ситуацию, когда $\mathscr{T}=\{\varnothing, T\}$. Нетрудно проверить, что в этом случае совокупность $\mathscr{T} \times \mathfrak{N}_{\varphi}$ является $\sigma$-алгеброй, так что $\mathscr{T} \otimes \mathfrak{N}_{\varphi}=\mathscr{T} \times \mathfrak{N}_{\varphi}$. Допустим, что каждое из множеств $T$ и $X$ содержит более одного элемента, и возьмем такую точку $\left(t_{0}, x_{0}\right) \in T \times X$, что $x_{0} \neq \varphi\left(t_{0}\right)$. Положим $D=\left\{\left(t_{0}, x_{0}\right)\right\}$. Тогда $D \in \mathfrak{M}_{\varphi}$, так как $G_{\varphi}^{-1}(D)=\varnothing \in \mathscr{T}$. В то же время $D \notin \mathscr{T} \times \mathfrak{N}_{\varphi}$, так как $\left\{t_{0}\right\} \notin \mathscr{T}$.

ЗАМЕЧАНИЕ 2. ПолОЖим

$$
J=\{A \in \mathscr{T}:(E \subset A) \Longrightarrow(E \in \mathscr{T})\} .
$$

Легко убедиться, что $J$ является $\sigma$-идеалом (т.е. $\varnothing \in J$, семейство $J$ счетно-аддитивно, и если $A \in J$, а $E \subset A$, то $E \in J$ ), причем максимальным из $\sigma$-идеалов (и даже из идеалов), содержащихся в $J$.

Например, если $T=I R$, а $\mathscr{T}$ - совокупность всех измеримых по Лебегу множеств в $I R$, то $J$ состоит из всех множеств меры нуль.

Пусть функции $\varphi, \psi \in X^{T}$. Назовем их $J$-эквивалентными (обозначение: $\varphi \stackrel{J}{\sim}$ $\psi)$, если $\{t \in T: \varphi(t) \neq \psi(t)\} \in J$. Легко убедиться, что отношение $J$ является отношением эквивалентности на множестве $X^{T}$.

Если $\varphi \stackrel{J}{\sim} \psi$ и $B \subset X$, то, как нетрудно проверить, $\varphi^{-1}(B) \in \mathscr{T}$ тогда и только тогда, когда $\psi^{-1}(B) \in \mathscr{T}$. Это значит, что $\mathfrak{N}_{\varphi}=\mathfrak{N}_{\psi}$. Аналогично, $\mathfrak{M}_{\varphi}=\mathfrak{M}_{\psi}$.

Отсюда следует, что при рассмотрении $\sigma$-алгебр $\mathfrak{N}_{\Phi}$ и $\mathfrak{M}_{\Phi}$ можно считать, что никакие две функции, входящие в $\Phi$, не являются $J$-эквивалентными.

ЗАмечание 3. Пусть $\Phi \subset X^{T}$. Известно (см., например, [4]) понятие склейки множества $\Phi$. Склейка $S(\Phi)$ состоит из всевозможных функций $\varphi: T \rightarrow X$, определяемых следующим образом. Возьмем произвольное счетное (в частности, конечное) разбиение $\left\{T_{n}: n \in I\right\}$ множества $T$ и какие-либо функции $\varphi_{n} \in \Phi, n \in I$. Тогда если $\varphi(t)=\varphi_{n}(t), t \in T_{n}, n \in I$, то $\varphi \in S(\Phi)$. 
Если при этом рассматриваются только те разбиения, у которых $(\forall n \in I) T_{n} \in \mathscr{T}$, то множество соответствующих функций $\varphi \in S(\Phi)$ обозначим через $S(\Phi, \mathscr{T})$.

Покажем, что $\mathfrak{M}_{S(\Phi, \mathscr{T})}=\mathfrak{M}_{\Phi}$ и $\mathfrak{N}_{S(\Phi, \mathscr{T})}=\mathfrak{N}_{\Phi}$. Действительно, так как $\Phi \subset$ $S(\Phi, \mathscr{T})$, то $\mathfrak{M}_{S(\Phi, \mathscr{T})} \subset \mathfrak{M}_{\Phi}$ и $\mathfrak{N}_{S(\Phi, \mathscr{T})} \subset \mathfrak{N}_{\Phi}$. Далее, пусть $B \in \mathfrak{N}_{\Phi}$, т.е. $(\forall \varphi \in \Phi)$ $\varphi^{-1}(B) \in \mathscr{T}$, и пусть $\psi \in S(\Phi, \mathscr{T})$, т.е. $\psi(t)=\varphi_{n}(t), t \in T_{n}, n \in I$, где $(\forall n) T_{n} \in \mathscr{T}$ и $\varphi_{n} \in \Phi$. Тогда

$$
\psi^{-1}(B)=\bigcup_{n \in I}\left(T_{n} \cap \varphi_{n}^{-1}(B)\right) \in \mathscr{T},
$$

так что $B \in \mathfrak{N}_{S(\Phi, \mathscr{T})}$. Аналогично получаем, что $\mathfrak{M}_{\Phi} \subset \mathfrak{M}_{S(\Phi, \mathscr{T})}$.

ЗАмечАниЕ 4 . Как мы видели, $\sigma$-алгебры $\mathfrak{M}_{\varphi}$ и $\mathfrak{N}_{\varphi}$ "обслуживают" условия $(\mathscr{T}, \mathscr{B})$-измеримости суперпозиций $f \circ G_{\varphi}$ и $g \circ \varphi$ соответственно. При этом речь идет об условиях на "внешние" функции $f$ и $g$. Естественно рассмотреть также условия на функцию $\varphi$ при фиксированных $f$ и $g$. В этих условиях, которые также вытекают из равенств (а) и (b), фигурируют $\sigma$-алгебры

$$
f^{-1}(\mathscr{B}):=\left\{f^{-1}(C): C \in \mathscr{B}\right\}
$$

$($ на $T \times X)$ и $g^{-1}(\mathscr{B})($ на $X)$. А именно, суперпозиция $f \circ G_{\varphi}$ (соответственно $g \circ$ $\varphi)(\mathscr{T}, \mathscr{B})$-измерима тогда и только тогда, когда функция $G_{\varphi}$ (соответственно $\left.\varphi\right)$ $\left(\mathscr{T}, f^{-1}(\mathscr{B})\right)$-измерима (соответственно $\left(\mathscr{T}, g^{-1}(\mathscr{B})\right)$-измерима).

\section{СПИСОК ЦИТИРОВАННОЙ ЛИТЕРАТУРЫ}

[1] И. В. Шрагин, "Условия измеримости суперпозиций”, Докл. АН СССР, 197:2 (1971), 295-298.

[2] И. В. Шрагин, "Суперпозиционная измеримость”, Изв. вузов. Матем., 1975, № 1, 8292.

[3] J. Appell, P.P. Zabrejko, Nonlinear Superposition Operators, Cambridge Univ. Press, Cambridge, 1990.

[4] Ю. В. Непомнящих, А. В. Поносов, “Локальные операторы в некоторых подпространствах пространства $L_{0}$ ", Изв. вузов. Матем., 1999, № 6, 50-64. 